\title{
Comprehensive Evaluation for Protective Coatings: Optical, Electrical, Photoelectrochemical, and Spectroscopic Characterizations
}

\author{
Xin Shen ${ }^{1,2}$, Rito Yanagi ${ }^{1,2}$, Devan Solanki ${ }^{1,2}$, Haoqing Su ${ }^{1,2}$, Zhaohan $\mathrm{Li}^{1,2}$, \\ Cheng-Xiang Xiang ${ }^{3 *}$ and Shu $\mathrm{Hu}^{1,2 *}$
}

${ }^{1}$ Department of Chemical and Environmental Engineering, School of Engineering and Applied Sciences, Yale University, New Haven, CT, United States, ${ }^{2}$ Energy Sciences Institute, Yale West Campus, West Haven, CT, United States, ${ }^{3}$ Division of Engineering and Applied Science, California Institute of Technology, Pasadena, CA, United States

\section{OPEN ACCESS}

Edited by:

Rahul R. Bhosale,

Qatar University, Qatar

Reviewed by:

Avner Rothschild,

Technion Israel Institute of

Technology, Israel

Shankara Kalanur,

Ajou University, South Korea

*Correspondence:

Cheng-Xiang Xiang

cxx@caltech.edu

Shu Hu

shu.hu@yale.edu

Specialty section:

This article was submitted to

Hydrogen Storage and Production,

a section of the journal

Frontiers in Energy Research

Received: 22 October 2021

Accepted: 17 December 2021

Published: 12 January 2022

Citation:

Shen X, Yanagi R, Solanki D, Su H, LiZ, Xiang C-X and Hu S (2022)

Comprehensive Evaluation for

Protective Coatings: Optical, Electrical,

Photoelectrochemical, and

Spectroscopic Characterizations.

Front. Energy Res. 9:799776.

doi: 10.3389/fenrg.2021.799776
Numerous efficient semiconductors suffer from instability in aqueous electrolytes. Strategies utilizing protective coatings have thus been developed to protect these photoabsorbers against corrosion while synergistically improving charge separation and reaction kinetics. Recently, various photoelectrochemical (PEC) protective coatings have been reported with suitable electronic properties to ensure low charge transport loss and reveal the fundamental photoabsorber efficiency. However, protocols for studying the critical figures of merit for protective coatings have yet to be established. For this reason, we propose four criteria for evaluating the performance of a protective coating for PEC water-splitting: stability, conductivity, optical transparency, and energetic matching. We then propose a flow chart that summarizes the recommended testing protocols for quantifying these four performance metrics. In particular, we lay out the stepwise testing protocols to evaluate the energetics matching at a semiconductor/coating/ (catalyst)/liquid interface. Finally, we provide an outlook for the future benchmarking needs for coatings.

Keywords: coating, performance evaluation, performance metrics, energetics, spectroscopy

\section{INTRODUCTION}

Energy conversion materials such as semiconductor photoabsorbers likely undergoe chemical, electrochemical, or photochemical corrosion during photoelectrochemical reductive or oxidative reactions (Chen and Wang, 2012; Zheng et al., 2019). Thus, protective coatings were developed to protect those otherwise unstable semiconductor photoabsorbers against corrosion (Walter et al., 2010; Paracchino et al., 2012; Hu et al., 2015; Gu et al., 2017). As the field evolved, stabilization coatings alone or in conjunction with co-catalysts have been developed to promote charge separation (Gu et al., 2016; Pan et al., 2019; Zhao et al., 2021), to improve charge transport kinetics, to passivate surface states (Le Formal et al., 2011), to form rectifying heterojunction (Scheuermann et al., 2016), and to boost surface reaction rates (Chen et al., 2020; Kawde et al., 2020; Pastukhova et al., 2021). However, the addition of coatings and co-catalysts creates new interfaces and new electronic states: one fundamental requirement is the energetic compatibility with the underlying photoabsorbers to reveal the photoabsorber fundamental performance and maximize solar-to-chemical conversion efficiency. Furthermore, the employment of coating is not limited to PEC water splitting, but a wider 

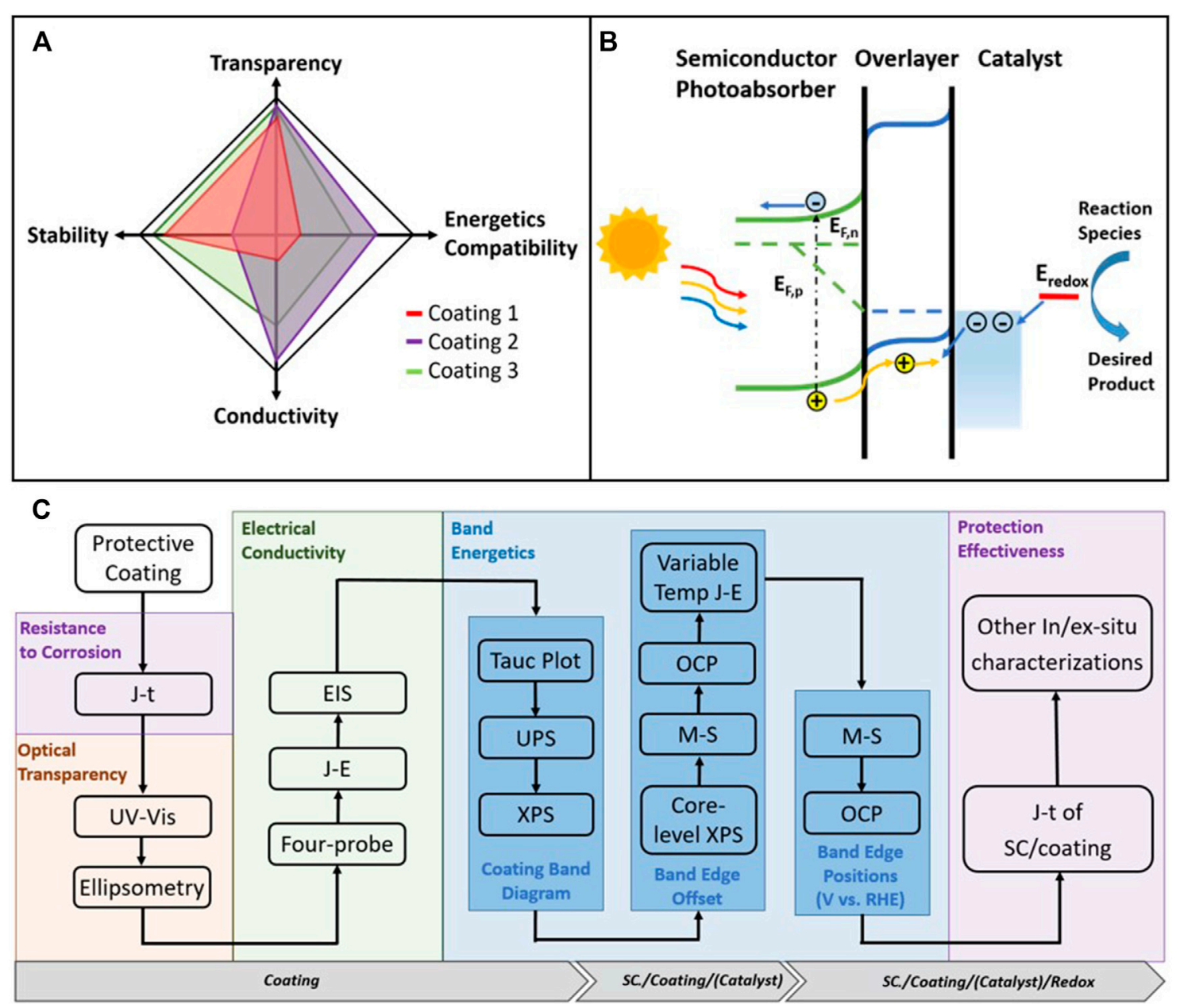

FIGURE 1 | (A) Illustration of the four performance metrics for evaluating protective coatings for PEC water-splitting devices. The performance metrics for three representative coatings are also shown. They include: stability (primarily lifetime/hours), optical transparency (primarily transmittance/\%), conductivity (primarily resistance/ohm) and energetics compatibility (primarily charge transfer efficiency across interface/\%); (B) Schematics for showing the concept of energetics compatibility (here, the photoanode case is used as an example); (C) Flow chart that summarizes the recommended testing protocols for quantitatively evaluating the four performance metrics of a protective coating. Abbreviations: current-time (J-t), current-potential (J-E), electrochemical impedance spectroscopy (EIS), ultraviolet photoelectron spectroscopy (UPS), X-ray photoelectron spectroscopy (XPS), Mott-Schottky (M-S), and open-circuit potential (OCP). SC stands for semiconductor.

fields such as dye sensitized PEC cells, perovskite solar cells, battery, and fuel cell devices, the materials and components of which have been reported with stability issues and require coating protection (Kay and Gratzel, 2002; Wang et al., 2008; Liu et al., 2015; Park and Zhu, 2020).

At the current stage, we recognize the challenges of comparing and evaluating the performance of coating strategies for different applications using a standardized approach. Protective coatings are often reported with various growth techniques and conditions and in combination with different underlying photoabsorbers and surface catalysts. The difficulty in deconvoluting the coating contribution from the overall performance of the coated device and interface (Hu et al., 2015). This results in a lack of data for the optical and electrical properties of the pure coating materials which hinder the progress of coating development. In addition, methods for evaluating device stability, coating stability, and optical properties are inconsistent among reports. In response to these inconsistencies, we present this work as an initiative to recommend comprehensive methodology for evaluating coating's performance.

Herein, we identify four performance metrics: stability, optical transparency, electrical conductivity, and energetics compatibility (Figure 1A) as the primary descriptors of protective coatings. In this paper, the concept of "stability" is two-fold: the coating's resistance to corrosion and its effectiveness at protecting the underlying photoabsorber. Resistance to corrosion includes both chemical and electrochemical resistances to corrosion, and it depends on the $\mathrm{pH}$ and local potentials. On the one hand, this photochemical stability criterion is firstly illustrated in a Pourbaix diagram (for electrochemical stability) for the thermodynamic corrosion potentials relative to the band edges (Chen and Wang, 2012; Hu et al., 2015). On the other hand, the stabilization efficiency (S), defined as the kinetic branching ratio of the local charge transfer current for desired reactions versus total 
light-induced current (Frese et al., 1981; Nandjou and Haussener, 2019), is often used to measure the coating's effectiveness for protecting the photoabsorber. The optical transparency is a unique requirement of the protective layer for sunlight driven processes. Any parasitic absorption and reflection from the protective layer would result in overall device efficiency loss. Depending on the detailed construct of the PEC cell, for example, the number of photoabsorbers and the bandgaps of the photoabsorbers, the requirement for the optical transparency could be very different. The electrical conductivity of a coating depends on in-plane and through-plane conductivity or resistance. The through-plane resistance consists of the contact resistance at the coating-absorber and the coating-liquid interfaces plus the coating bulk resistance, all of which need to be minimized to reduce the potential loss. Energetic compatibility is another critical aspect of protective coatings. It is achieved when the energy levels of charge carriers on both the photoabsorber side and the liquid interface side of the coating align, as shown in Figure 1B. The matching minimizes the energy barrier and the energy loss for charge transfer across the interface. Even though the photoabsorber and the protective layer may have excellent properties by themselves, a mismatch in the chargetransport energetic levels between the two can result in very low conversion efficiency.

While ideal coatings are expected to excel in all four categories, there are often inherent trade-offs when optimizing their properties. For example, conductivity often comes at the expense of transparency and stability: wide-bandgap oxides are usually more optical transparent and stable than narrow bandgap materials but at the cost of being more electrically insulating. The reason is that the electronic states for transporting charges through the coating and achieving energetic matching are often missing or lower in the density of states than narrow bandgap materials (Frese et al., 1980; Sze and Ng, 2007). One strategy to improve the conductivity of wide-bandgap oxide materials without sacrificing stability and optical transparency is to introduce intermediate band (IB) or in-gap defect states (Hu et al., 2016). Recently, methods for engineering intermediate bands (IB) or defect states in wide bandgap coatings avoid these trade-offs and allow for good transparency without sacrificing charge transport performance (Campet et al., 1989; Hu et al., 2014; Bein et al., 2019; Li et al., 2021). These coatings modify the interfacial thermodynamics, carrier dynamics, and surface reaction pathways, which require special characterization techniques to elucidate (Dai et al., 2020). This paper summarizes these characterization techniques and their measurement approaches to illustrate these coating characterization protocols.

To illustrate the application of the protocol, we consider three representative coatings and their corresponding four performance metrics as examples (Figure 1). Tunneling $\mathrm{TiO}_{2}$ overlayers developed for metal-insulator-semiconductor (MIS) $\mathrm{Si}$ photoanode are illustrated as Figure 1A coating 1 (Chen et al., 2011; Scheuermann et al., 2013). This approach stabilized heavily $\mathrm{p}^{+}$-doped Si for $8 \mathrm{~h}$ under both acidic and basic conditions (Lin et al., 2013). Despite the optical transparency of few-nanometer $\mathrm{TiO}_{2}$, the energetic mismatch and low conductivity of this $\mathrm{TiO}_{2}$ coating resulted in $\sim 21 \mathrm{mV}$ of added overpotentials at $1 \mathrm{~mA} \mathrm{~cm}^{-2}$ per nanometer of $\mathrm{TiO}_{2}$ for thicknesses greater than $\sim 2 \mathrm{~nm}$. The primary mechanism of charge transport through this coating is by charge tunneling, as its energetics is incompatible with the photoabsorber and the water oxidation electrocatalyst. $\mathrm{NiO}_{\mathrm{x}}$ (Sun et al., 2015c), illustrated as Figure 1A coating 2, was shown to stabilize Si photoanodes in $1 \mathrm{M} \mathrm{KOH}(\mathrm{aq})$ for $5 \mathrm{~h}$. The long-term stability of $\mathrm{NiO}_{\mathrm{x}}$-protected $\mathrm{Si}$ photoanodes was inferior relative to other wide bandgap oxide coatings because the $\mathrm{NiO}_{\mathrm{x}}$ layer underwent microstructure changes after redox cycling, which made the $\mathrm{NiO}_{\mathrm{x}}$ layer ion-permeable (Lin and Boettcher, 2014). Despite that, $\mathrm{NiO}_{\mathrm{x}}$ has its own strength in high transparency, metal-like conductivity, and negligible energy loss for the various efficient photoabsorbers performing lightinduced charge transfer (Sun et al., 2015a; Sun et al., 2015b; Sun et al., 2015c). "Leaky" $\mathrm{TiO}_{2}$ (Hu et al., 2014) (Figure 1A: coating 3) with $\mathrm{Ni} / \mathrm{NiO}_{\mathrm{x}}$ electrocatalysts is stable in $1 \mathrm{M} \mathrm{KOH}(\mathrm{aq})$ for over thousands of hours (Shaner et al., 2015) and has an average 80\% transmission in the visible light range (Hu et al., 2014). In this coating/co-catalyst combination, the $\mathrm{TiO}_{2}$ has nearly thicknessindependent hole-transport conductivity which is four orders of magnitude higher than the insulator $\mathrm{TiO}_{2}$ reported above (Scheuermann et al., 2013; Scheuermann et al., 2016), and is energetically compatible with photoabsorbers such as $\mathrm{Si}$ and CdTe (Hu et al., 2014; Chen et al., 2020). So far, a few protective layers including the "leaky" $\mathrm{TiO}_{2}$ achieve the stablility, transparency, conductivity, and the proper energetic matching for several technologically important photoabsorbers of $<1.4 \mathrm{eV}$ bandgaps. But further improvement is anticipated to further broaden coatings' practice with photoabsorbers of $>1.7 \mathrm{eV}$ bandgaps and enhancing its corrosion resistance and protection effectiveness.

We recommend a standard procedure to evaluate these four metrics, as shown in the flow chart Figure 1C. Quantification of the stability (primarily lifetime/hours), optical transparency (primarily transmittance/\%), conductivity (primarily resistance/ohm) and energetics compatibility (primarily charge transfer efficient accorss interface/\%) under the control of other aspects such as thickness and substrate, help to evaluate protective coating comprehensively and highlights the current deficiencies and constraints. Such an evaluation can help create guidelines for designing and developing more efficient and multifunctional coating materials. We note that corrosion resistance is a prerequisite for a coating material. Therefore, it is the first aspect to be investigated, while the remaining four criteria can be studied based on a specific application.

\section{Stability}

Both the coating's resistance to corrosion and its effectiveness at protecting the PEC device should be measured. However, in many articles, only the time-dependent current or potential behavior, which measures the effectiveness, is reported according to the PEC test protocol used in the last decade (Chen et al., 2013a). While stable device operation should imply coating's resistance to corrosion, an explicit study is still valuable, because 1) it is a primary screening tool and a prerequisite for developing a new coating (Siddiqi et al., 2018); 2 ) it helps distinguish the pitting corrosion of the underlying 
protected photoabsorber from the dissolution of the coating (Shen et al., 2021); and 3) it helps better understand the corrosion or failure mechanism of the coating stabilized interface (Gerischer, 1977; 1991). For testing the coating's resistance to corrosion, it is typical to grow coatings on conductive substrates initially and use electrochemistry methods. These methods include chronoamperometry to observe the current degradation and chronopotentiometry to test the potential deviation (Moehl et al., 2017; Siddiqi et al., 2018), in combination with various in-situation or ex-situ characterizations, such as XPS to compare the chemical change and electronic structure change after the test with those before (Moehl et al., 2017; Siddiqi et al., 2018).

For evaluating the effectiveness of protection, the porosity as part of the ion permeability test (Jung et al., 2018b) can be used for measuring how effectively the coating reduces the diffusion of reactive species from an electrolyte solution. Since the protection effectiveness directly reflects the operational stability, electrochemical methods are usually used to test the coated PEC device's operational lifetime, sometimes even imitating the practical conditions (e.g., biased, AM 1.5 one Sun illumination, neutral $\mathrm{pH}$, simulated diurnal cycles, etc.). Thus, they are powerful and indispensable tools for studying a coating's stability as they consider realistic operating conditions for photoelectrochemistry applications.

The evaluation of device operational stability can be short term or long term. While short-term tests are primarily conducted under harsh conditions for examining the robustness of the coated photoabsorber and studying their corrosion mechanism, long-term tests are employed to demonstrate practical viability (Vanka et al., 2019). As the operational lifetime test alone cannot reveal the compositional or structural changes during operation, these tests are typically combined with a series of compositional, morphological, and topographical characterizations (both in-situ and ex-situ). Characterization techniques include morphological: AFM and ac mode AFM (for local metastable species) (Cheng et al., 2017; Ros et al., 2019; Vanka et al., 2019), Cross-section SEM (structural integrity) (Yu et al., 2018), TEM (nanoscale integrity) (Cheng et al., 2017); compositional: SEM-EDS (surface mapping of the composition) (Shen et al., 2021), XPS (surface atoms oxidation state change) (Pishgar et al., 2019; Cao et al., 2020), ICP-MS (Materials dissolution) (Pishgar et al., 2019).

\section{Optical Properties}

A comprehensive optical characterization of a protective coating includes the study of both the intrinsic properties, such as the dielectric constants $(n, k)$ and the extrinsic properties, such as absorption, transmission, and reflection. Those optical characteristics depend on the coating's thickness and morphology.

The dielectric properties $(n, k)$ of thin films can be determined by Ellipsometry which measures the change in polarization as light reflects or transmits from the sample. The polarization change is represented as an amplitude ratio and the phase difference. The measured complex dielectric constants are the thickness-independent optical property of a coating. They are closely related to the dopant concentration and oxidation states, and thus also serve as an important input parameter for optical simulations (e.g., Finite-Difference Time-Domain modeling) (Mohsin et al., 2020). Ellipsometry is an indirect method, where the $(n, k)$ values are obtained by fitting the measured light amplitude ratio and the phase difference with a dielectric function model. Therefore, selecting the appropriate model based on the material type and wavelength used for analysis is crucial for obtaining meaningful results. For anisotropic or inhomogeneous coating materials, the Mueller matrix formalism should be used to account for depolarization (Fujiwara, 2007).

The extrinsic optical properties are commonly characterized by ultraviolet-visible spectroscopy (UV-Vis). Initial screening for low optical loss involves measuring the transmission, reflection and absorption spectra of a substrate with/without coating for comparing the effect of coating (Sun et al., 2015c). The coating reflectance is recommended to be measured by either diffuse reflectance spectroscopy (DRS) using an integrating sphere (when the interference effect need to be rule out), or specular reflectance (when the interference effect need to be taken into account) (Chen et al., 2013b). While the UV-Vis solid film measurement is conducted in air, it is most relevant for reflectance measurements when the coated sample is immersed in liquids, so the measurement responds to the PEC operational environment. Lastly, the absorption spectra can also be used to construct a Tauc plot for measuring the optical bandgap, which is crucial information of the energy band diagram (Makula et al., 2018).

It should be noted that the thin-film interference effect may influence the reflectance, transmittance, and absorption of the coated photoelectrode. If the coating thickness is comparable to the incident light wavelength and the phase delay between the reflected light at two interfaces of the thin film module as a function of coating thickness, the reflected and transmitted light intensity will constructive and destructive interfere alternatively (Wolter, 1966). The use of Ellipsometry to measure dielectric $(\mathrm{n}, \mathrm{k})$ properties elucidates the interference issue. Coatings should be compared at the same thickness outside of the interference regime (Kats et al., 2014; Xu et al., 2018). This way avoids the inaccurate assessment caused by this effect and compares the coating extrinsic optical properties fairly. In some cases, the surface morphology (roughness or special surface patterns) can also contribute to the UV-Vis spectra, and therefore this factor should be considered when benchmarking the coating optics (Xu et al., 2018).

\section{Electrical Conductivity}

The methods for characterizing in-plane and through-plane conductivity of bulk coatings include: 1) Electrochemical Impedance Spectroscopy (EIS), which quantitatively measures the through-plane conductivity. The precision of the EIS technique depends on the selection and fitting of equivalent circuits. Besides the coating through-plane resistance, a typical equivalent circuit also consists of solution series resistance, charge-transfer resistance, and space-charge and surface state capacitances in series or parallel. Given the multiple fitting 
parameters in the equivalent circuits, it is important to ensure all parameters have physical meanings. The selection accuracy and model validation can be referred to by Spyker and coworkers (Jiya et al., 2018); 2) Electrochemical cyclic voltammetry at the low bias region for through-plane conductivity, in which the resistance can be derived from the extrapolation of the linear region at low bias (Nunez et al., 2019). It should be noted that this method only applies when the coating resistance dominates the through-layer resistance; 3) Sheet resistance for in-plane conductivity, which can be measured by the four-point probe method (Hu et al., 2014); 4) Hall measurement for in-plane conductivity, a technique that employs a magnetic field perpendicular to the in-plane current flow, can be used to measure charge carrier density, electrical resistance, and carrier mobility for the bands of the films from which the conductivity can be derived ( $\mathrm{Hu}$ et al., 2014). The through-plane resistance can limit the coating thickness selection, and therefore indirectly affect the stability and optical transparency of the device. Here, one typically assumes that coating conductivity is isotropic, which can be validated by the comparison of in-plane and through-plane resistivity.

In addition to providing information about coating resistance, conductivity studies can also be useful for revealing charge conduction mechanisms for the coating by: 1) solid-state I-V measurement of coatings deposited on the substrates of varying work functions; 2) alternating current-conductance of the through-layer device as a function of frequency under a fixed temperature and fixed applied bias, where the response can be used to fit the model for band-mediated charge transport, or model for charge transport via hopping, therefore help determine the charge transport pathway; 3) temperature-dependent direct current conductance. This temperature-dependent conductance can be used to determine the activation energy, which reveals the mechanism of tunneling or hopping based on charge transfer barrier and conduction mechanism (Nunez et al., 2019); 4) spacecharge-limited current spectroscopy, where the conductivity of the coating in contact with the solution is measured over a range of gate potentials (Nunez et al., 2019). The potential-dependent conductivity is done by interdigitated electrodes in a field-effect transistor configuration, where I-V conductivity is measured with the varying Fermi-energy level (applied gate voltages) (Roest et al., 2002; Plana et al., 2013). This technique is also commonly used for studying the conduction mechanism in combination with the frequency and temperature varied conductance measurement (Nunez et al., 2019). The above techniques mainly focus on the macroscopic electrical properties of the coating. Last but not the least, conductive atomic force microscopy (c-AFM) can be used for testing local conductivity on the film surface or the cross-section for revealing coating inhomogeneity or detecting metastable phases (Yu et al., 2018; Ros et al., 2019).

\section{Energetics Compatibility}

The interfaces between the protective coating and the photoabsorber, with or without surface-attached catalysts (Walter et al., 2010; Hu et al., 2015; Bae et al., 2017) are vital for thermodynamics, charge separation, charge transfer and reaction kinetics (Thorne et al., 2015; Vanka et al., 2019). The protective coating's electrical properties should be tailored to the photoabsorber and the catalyst for minimizing energy conversion loss. In doing so, the band bending and defect band alignment across the SC/coating interface can be tailored towards efficient charge transfer, thus allowing for thicker protective coatings and eliminating manufacturing defects. Without proper energetics compatibility, charge transport through SC/coating interface would primarily rely on charge tunneling. Hence, the coating thickness is constrained to $<3 \mathrm{~nm}$ (Scheuermann et al., 2016), which makes the protective coating prone to degradation.

Since energetics depends on interfacial chemistry, it is imperative to standardize surface treatment before the coating deposition to remove surface oxide and achieve reproducible substrate/coating interface conditions. Procedures for surface treatment requires the selection and standardization of photoabsorbers to ensure fair evaluation. For example, in the case of $\mathrm{n}^{+} \mathrm{Si} / \mathrm{TiO}_{2}$, the $\mathrm{Si}$ substrate has a well-established surface treatment protocol: an RCA SC-1 etch, followed by immersion in $5 \mathrm{M}$ hydrofluoric (HF) acid, and an RCA SC-2 procedure (Hu et al., 2014). This series of surface treatments produce a $\mathrm{SiO}_{2}$ tunnel interface, which passivates surface states responsible for non-radiative carrier recombination and aligns the $\mathrm{Ti}^{3+}$ defect band to the Si valence band.

Determination of the complete band energetic diagram of the coated PEC device follows a protocol with three major steps, each requiring a series of characterizations as illustrated in Figure 2. The first step (Figures 2A,B) for mapping band energetics is to measure the coating bulk band diagram in vacuum. Tauc plot ( $h v-\alpha h v$ plot) by UV-Vis can be used to determine the optical band gap (Figure 2A). The film absorption coefficient $a$ is measured as mentioned in the optical measurement section. Extrapolation of the linear region of the hv $-(\alpha h v)^{1 / 2}$ plot yields the energy of the optical bandgap of the amorphous material ( $\mathrm{Hu}$ et al., 2014; Siddiqi et al., 2018). Alternatively, the electronic bandgap of some ultra wide bandgap oxides, such as $\mathrm{SiO}_{\mathrm{x}}$ can be obtained by high energy XPS using the difference in energy between the elastic peak (e.g., oxygen peak) and the onset of inelastic losses (Nichols et al., 2014; Iatsunskyi et al., 2015). Ultraviolet photoelectron spectroscopy (UPS) can provide information including the work function, i.e., the Fermi level position versus the vacuum potential, and the surface density of states (Hu et al., 2014; Hu et al., 2016; Richter et al., 2021).

Both UPS and valence XPS can be used for determining the band edge position relative to the Fermi level. However, while UPS only probes the film's surface, valence XPS provides a more accurate means to analyze the bulk film's valence band position because the greater penetration depth of the XPS probe beam reduces the effect of spurious surface states during the analysis of the valence band position (Lichterman et al., 2015; Hu et al., 2016; Richter et al., 2021). For the valence XPS measurement, the coating sample surface needs to be partially covered by a gold foil and grounded to the stage. The scan range typically starts from -5 to $20 \mathrm{eV}$ vs. $0 \mathrm{eV}$ binding energy, defined as the Fermi level. The conductivity of the film sample needs to be ensured to avoid the electron charging effect, with the valence spectra of gold 


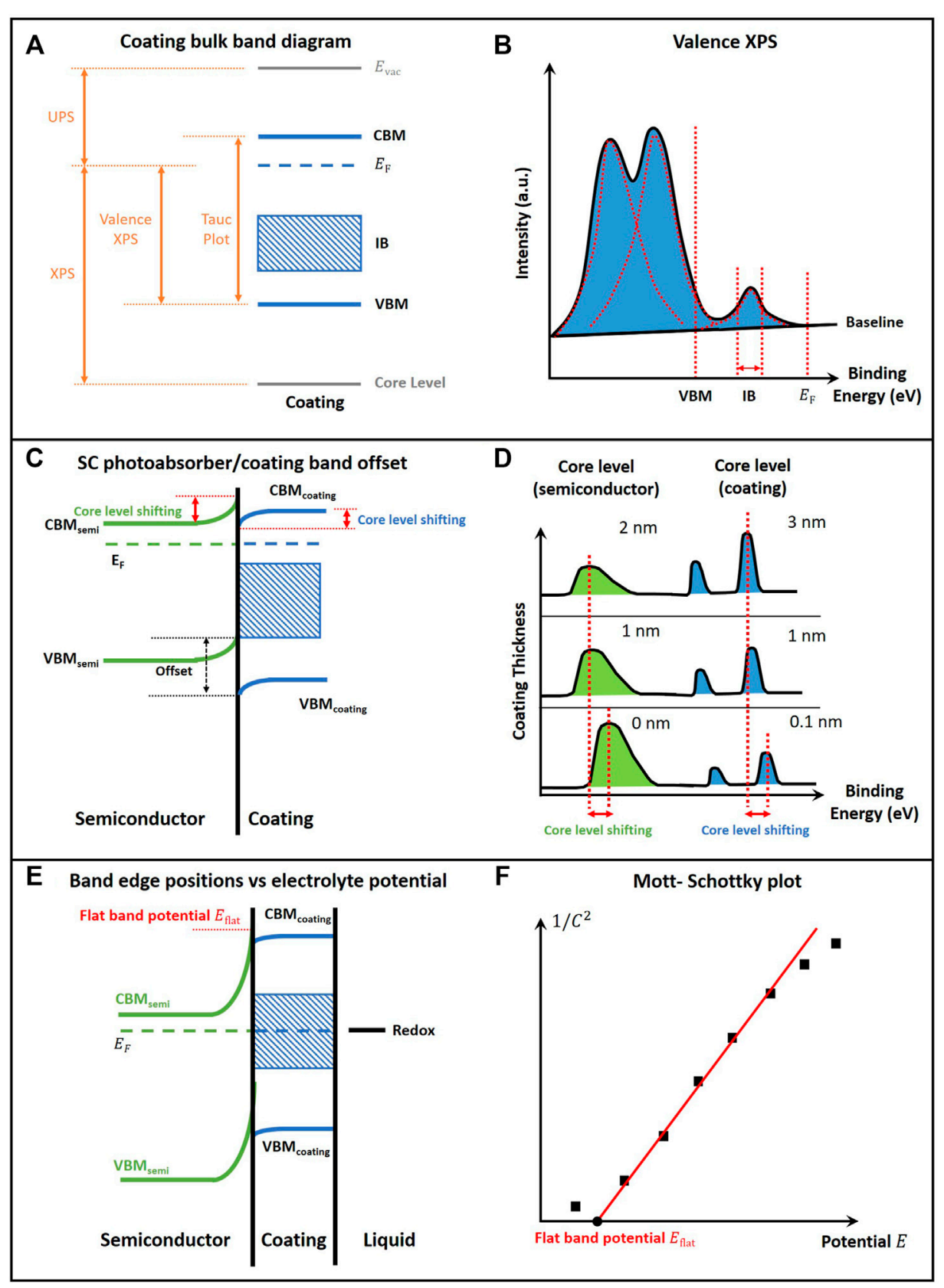

FIGURE 2 | The development of band-energy diagram of semiconductor photoabsorber/coating/(catalyst)/liquid electrolyte. (A) Band-energy diagram for bulk coating; (B) Valence XPS data with peak deconvolution results; (C) Band-energy diagram for semiconductor photoabsorber/coating integrace, which can be obtained from literature report, and the measured position of the peak from XPS at the interface (as indicated by the red arrow); (D) The characteristic XPS core level peak shifting for the semiconductor and the coating; (E) Band-energy diagram for semiconductor photo absorber/coating/(catalyst)/liquid; (F) Mott-Schottky plot, i.e., the reciprocal of the square of capacitance versus the potential between the bulk electrode and the bulk electrolyte.

or palladium measured as a reference for the binding-energy calibration (Sharpe et al., 2017).

Peak deconvolution of the raw valence spectra data can be achieved by software such as Casa XPS or Multipak. The assignment of the characteristic peaks should follow the previous report of the electronic study of the material or its component. The result of the valence XPS allows for the determination of the coating energy levels (including $\mathrm{CB}, \mathrm{VB}$, and IB) relative to the Fermi level of the material, while UPS determines the valence band position and the work function. This step completes the derivation of the energetics of bulk coating. The valence band edge (VBM) is determined by linear extrapolation of the characteristic peak. The intermediate band center position and width corresponds to the peak position of the 
intra gap electronic states, and its FWHM of measured XPS peaks. After the peak deconvolution analysis of valence XPS spectra and UPS (Figure 2B), a bulk energy diagram can be constructed (Figure 2A).

The second step (Figures 2C,D) for mapping band energetics is to derive the band edge offset between the SC photoabsorber and the coating. When a coating is applied to a semiconductor, the degree of band bending can be determined by comparing the shift of the core-level XPS peak positions for the samples with incremental increases of coating thickness (Klein et al., 2008; Hu et al., 2016; Richter et al., 2021). Usually, overlayer thicknesses of over $4 \mathrm{~nm}$ are not recommended because they block the substrate signal due to the limits of penetration depth or mean free path of the photoelectrons, unless using hard X-ray spectroscopy. The characteristic core-level XPS spectra can be measured for both the substrate and the coating. For example, in the case of $\mathrm{Si} / \mathrm{TiO}_{2}$, Si$2 p$ core-level spectra can be measured for deriving the Si band bending, while the Ti-2p spectra can be obtained for the band bending on the protective coating. The magnitude of band bending is determined by the shift of the characteristic positions of the core-level peak that belong to the semiconductor photoabsorber, as shown in Figure 2D. For the protective coating, the band bending can be derived in the same manner by the binding energy difference between the bulk (when coating thickness is $>2 \mathrm{~nm}$ ) and interface (when the coating is only few cycles by atomic layer deposition) (as indicated by the red arrow) (Klein et al., 2008; Hu et al., 2016). Assuming that the Fermi level of semiconductor and coating align under equilibrium in the dark, the band offset can be determined in conjunction with the characterization of band edge positions relative to the Fermi level. Then, the band bending values for both the semiconductor photoabsorber and coating can be obtained. An alternative option to obtain the band bending (barrier height) of the semiconductor photoabsorber is by the solid-state variable temperature $\mathrm{J}-\mathrm{V}$ measurements (Hu et al., 2016), or light-dependent opencircuit potential (OCP) measurements (Chen et al., 2013b; Dai et al., 2020), or by Mott-Schottky analysis (Hu et al., 2016). These techniques are often combined to provide a full picture of the band energetics and to validate one another.

The third step (Figures 2E,F) for mapping the band energetics is to determine the band edge positions of coatings in contact with the electrolyte solution of interest. We assume that the band offset at the semiconductor/coating solid-solid interface is independent of the contacting electrolyte (Tan et al., 1994; Walter et al., 2010). For the semiconductor/coating/liquid interface, Mott-Schottky (M-S) analysis can be used for obtaining the flat band potential and band edge positions (Figures 2E,F) (Gelderman et al., 2007; Hankin et al., 2019; Chen et al., 2020) By using the M-S analysis, the entire energy band diagram with respect to electrolyte potential can be mapped. Light-dependent OCP is an alternative option for determining the flat-band potential, where the Fermi level approaching the flat-band potential at high light intensity (Hankin et al., 2019).

The open-circuit photovoltage, measured from the difference between the dark and light OCP, in various redox electrolytes can also help to study the junction type (Hu et al., 2016). For PEC application, a buried junction with a fixed barrier height is often desired. In this case, the barrier height of the semiconductor junction depends on doping and built-in potential. This junction energetics have been fully exploited to achieve the desired performance independent of local $\mathrm{pH}$ environment and redox potentials (Dai et al., 2014; Chen et al., 2015; Hu et al., 2015).

These are cases that when semiconductor/coating get immersed in liquids, the band edge positions of coating and semiconductor with respect to liquid potential shift together with the applied bias and liquid potential. In this case, the band offset at the solid-solid interface remains constant with a fixed barrier height. When surface states dominate, the band edge of the solid will not be fixed under applied bias or light illumination (Jung et al., 2018a). This case occurs when surface states dominate. Inserting a coating between photoabsorbers and liquids can mitigate the Fermi level pinning, but for some porous and ionpermeable coatings, partial Fermi-level pinning can occur (Lin and Boettcher, 2014). A "dual-working-electrode" (DWE) technique can be employed to measure the local surface potential of the catalyst (Lin and Boettcher, 2014; Nellist et al., 2016), or a sophisticated electrochemical atomic force microscopy approach for electrochemical potential sensing can be employed (Nellist et al., 2018; Laskowski et al., 2020).

The aforementioned steps allow the construction of a complete band diagram of a coating protected semiconductor photoelectrode. The following characterizations are optional, used to validate the band energetics, providing energetics at operando conditions or for a local region. These techniques include in situ or operando XPS, which provide a band energy diagram at applied bias condition (Lichterman et al., 2015); Operando AFM which provides information including local site potential and carrier dynamics (Nellist et al., 2018; Connor et al., 2020; Kalanur et al., 2021); Resonant X-ray Photoelectron Spectroscopy (ResPES) and Resonant X-ray Spectroscopy (RiXS) can be used to quantify the energetics between the coating/catalyst interface that is immersed in liquids (Richter et al., 2021). Besides, ResPES and RiXS measurements were reported to be helpful for a thick catalyst layer since they can penetrate the relatively thick metal layer and examine the VB states using resonant excitation of a particular element.

In addition to the four metrics we mentioned in the former paragraph, we also acknowledge that the other aspect of coating should be taken into account for a comprehensive and fair evaluation. For example, as we mention in the introduction, coating can form a $\mathrm{p}$-n hetero-junction with the underlying absorber to promote the charge separation, therefore a desired coating candidate should have the opposite dopant type with appropriate doping concentration to form favorable band bending toward the corresponding surface reactions (Yang et al., 2019). Lastly, doped coating materials such as $\mathrm{Fe}$ doped $\mathrm{TiO}_{2}$, can contains trap defects that quench the photo-generated carriers before they migrate to the coating surface. The non-radiative recombination through coating-absorber interfaces and charge-transport 
states should be minimized not to affect charge separation (Kautek et al., 1980; Singh et al., 2008).

\section{CONCLUSION AND PERSPECTIVE}

We recommended using the four metrics of stability, optical transparency, conductivity, energetics compatibility, and a series of protocols to evaluate protective coating materials systematically. These metrics can also serve as a helpful guide for the coating design and comparing performance across different coatings. We summarized the current methods reported in the literature for evaluating these four metrics, along with common pitfalls. Lastly, we discussed a stepwise procedure for deriving the complete band energy diagram for a complex SC photoabsorber/coating/liquid interface with or without catalysts.

We note that there are challenges that require further research, such as the inherent trade-off between the four metrics, as well as tailoring the intermediate-band states to specific needs. More advanced but less frequently utilized methods such as 1) comprehensive property observation under operando condition; 2) high throughput methods for screening and optimizing coating composition; 3) accelerated stress testing (AST) and corrosion mechanism study, which correlates the

\section{REFERENCES}

Bae, D., Seger, B., Vesborg, P. C. K., Hansen, O., and Chorkendorff, I. (2017). Strategies for Stable Water Splitting via Protected Photoelectrodes. Chem. Soc. Rev. 46, 1933-1954. doi:10.1039/c6cs00918b

Bein, N. S., Machado, P., Coll, M., Chen, F., Makarovic, M., Rojac, T., et al. (2019). Electrochemical Reduction of Undoped and Cobalt-Doped $\mathrm{BiFeO} 3$ Induced by Water Exposure: Quantitative Determination of Reduction Potentials and Defect Energy Levels Using Photoelectron Spectroscopy. J. Phys. Chem. Lett. 10, 7071-7076. doi:10.1021/acs.jpclett.9b02706

Campet, G., Puprichitkun, C., and Sun, Z. W. (1989). Protection of Photoanodes against Photocorrosion by Surface Deposition of Oxide Films. J. Electroanalytical Chem. Interfacial Electrochemistry 269, 435-445. doi:10.1016/0022-0728(89)85150-2

Cao, S. Y., Kang, Z., Yu, Y. H., Du, J. L., German, L., Li, J., et al. (2020). Tailored $\mathrm{TiO} 2$ Protection Layer Enabled Efficient and Stable Microdome Structured P-GaAs Photoelectrochemical Cathodes. Adv. Energ. Mater. 10. doi:10.1002/ aenm.201902985

Chen, L., Yang, J., Klaus, S., Lee, L. J., Woods-Robinson, R., Ma, J., et al. (2015). p-Type Transparent Conducting Oxide/n-type Semiconductor Heterojunctions for Efficient and Stable Solar Water Oxidation. J. Am. Chem. Soc. 137, 9595-9603. doi:10.1021/jacs.5b03536

Chen, S., and Wang, L.-W. (2012). Thermodynamic Oxidation and Reduction Potentials of Photocatalytic Semiconductors in Aqueous Solution. Chem. Mater. 24, 3659-3666. doi:10.1021/cm302533s

Chen, X., Shen, X., Shen, S., Reese, M. O., and Hu, S. (2020). Stable CdTe Photoanodes with Energetics Matching Those of a Coating Intermediate Band. ACS Energ. Lett. 5, 1865-1871. doi:10.1021/ acsenergylett.0c00603

Chen, Y. W., Prange, J. D., Dühnen, S., Park, Y., Gunji, M., Chidsey, C. E. D., et al. (2011). Atomic Layer-Deposited Tunnel Oxide Stabilizes Silicon Photoanodes for Water Oxidation. Nat. Mater. 10, 539-544. doi:10.1038/ nmat 3047

Chen, Z., Dinh, H. N., and Miller, E. (2013a). Photoelectrochemical Water Splitting. Springer. coating permeability with the coating protection effectiveness, can further be developed following the protocols stated in this paper.

\section{AUTHOR CONTRIBUTIONS}

$\mathrm{C}-\mathrm{XX}$ and $\mathrm{SH}$ conceived the idea and designed this review. XS collected and reviewed the related literatures. XS and RY designed and made the schematics and figures with help from DS and HS. XS and DS wrote the manuscript. XS revised the manuscript with help from SH, CX and DS. SH revised the manuscript and oversaw the project. All authors contributed to the manuscript and provided feedback.

\section{FUNDING}

SH gratefully acknowledges the financial support of the National Science Foundation Award No. CBET-2055416. C-XX gratefully acknowledges the support from the Fuel Cell Technologies Office, of the United States Department of Energy, Energy Efficiency and Renewable Energy (EERE) under contract number DEEE0008092. RY acknowledges fellowship support from Japan Student Services Organization.

Chen, Z., Dinh, H. N., Miller, E., and Springerlink (Online Service (2013b). "Photoelectrochemical Water Splitting Standards, Experimental Methods, and Protocols," in SpringerBriefs in Energy.

Cheng, Q., Benipal, M. K., Liu, Q., Wang, X., Crozier, P. A., Chan, C. K., et al. (2017). Al2O3 and $\mathrm{SiO} 2$ Atomic Layer Deposition Layers on $\mathrm{ZnO}$ Photoanodes and Degradation Mechanisms. ACS Appl. Mater. Inter. 9, 16138-16147. doi:10.1021/acsami.7b01274

Connor, P., Schuch, J., Kaiser, B., and Jaegermann, W. (2020). The Determination of Electrochemical Active Surface Area and Specific Capacity Revisited for the System MnOx as an Oxygen Evolution Catalyst. Z. Physikalische ChemieInternational J. Res. Phys. Chem. Chem. Phys. 234, 979-994. doi:10.1515/zpch2019-1514

Dai, P., Li, W., Xie, J., He, Y., Thorne, J., Mcmahon, G., et al. (2014). Forming Buried Junctions to Enhance the Photovoltage Generated by Cuprous Oxide in Aqueous Solutions. Angew. Chem. Int. Ed. 53, 13493-13497. doi:10.1002/ anie. 201408375

Dai, Y., Yu, J., Cheng, C., Tan, P., and Ni, M. (2020). Engineering the Interfaces in Water-Splitting Photoelectrodes - an Overview of the Technique Development. J. Mater. Chem. A. 8, 6984-7002. doi:10.1039/d0ta01670e

Frese, K. W., Madou, M. J., and Morrison, S. R. (1980). Investigation of Photoelectrochemical Corrosion of Semiconductors. 1. J. Phys. Chem. 84, 3172-3178. doi:10.1021/j100461a008

Frese, K. W., Madou, M. J., and Morrison, S. R. (1981). Investigation of Photoelectrochemical Corrosion of Semiconductors: II . Kinetic Analysis of Corrosion-Competition Reactions on. J. Electrochem. Soc. 128, 1527-1531. doi:10.1149/1.2127676

Fujiwara, H. (2007). Spectroscopic Ellipsometry: Principles and Applications. John Wiley \& Sons.

Gelderman, K., Lee, L., and Donne, S. W. (2007). Flat-band Potential of a Semiconductor: Using the Mott-Schottky Equation. J. Chem. Educ. 84, 685-688. doi:10.1021/ed084p685

Gerischer, H. (1977). On the Stability of Semiconductor Electrodes against Photodecomposition. J. Electroanalytical Chem. Interfacial Electrochemistry 82, 133-143. doi:10.1016/s0022-0728(77)80253-2

Gerischer, H. (1991). Photodecomposition of Semiconductors - a Thermodynamic Approach - a Citation-Classic Commentary on the Stability of Semiconductor 
Electrodes against Photodecomposition by Gerischer,H. Berlin: Current Contents/Physical Chemical \& Earth Sciences, 10.

Gu, J., Aguiar, J. A., Ferrere, S., Steirer, K. X., Yan, Y., Xiao, C. X., et al. (2017). A Graded Catalytic-Protective Layer for an Efficient and Stable Water-Splitting Photocathode. Nat. Energ. 2. doi:10.1038/nenergy.2016.192

Gu, J., Yan, Y., Young, J. L., Steirer, K. X., Neale, N. R., and Turner, J. A. (2016). Water Reduction by a P-GaInP2 Photoelectrode Stabilized by an Amorphous TiO2 Coating and a Molecular Cobalt Catalyst. Nat. Mater. 15, 456, 460+ .doi:10.1038/nmat4511

Hankin, A., Bedoya-Lora, F. E., Alexander, J. C., Regoutz, A., and Kelsall, G. H. (2019). Flat Band Potential Determination: Avoiding the Pitfalls. J. Mater. Chem. A. 7, 26162-26176. doi:10.1039/c9ta09569a

Hu, S., Lewis, N. S., Ager, J. W., Yang, J., Mckone, J. R., and Strandwitz, N. C. (2015). Thin-Film Materials for the Protection of Semiconducting Photoelectrodes in Solar-Fuel Generators. J. Phys. Chem. C 119, 24201-24228. doi:10.1021/acs.jpcc.5b05976

Hu, S., Richter, M. H., Lichterman, M. F., Beardslee, J., Mayer, T., Brunschwig, B. S., et al. (2016). Electrical, Photoelectrochemical, and Photoelectron Spectroscopic Investigation of the Interfacial Transport and Energetics of Amorphous TiO2/ Si Heterojunctions. J. Phys. Chem. C 120, 3117-3129. doi:10.1021/ acs.jpcc.5b09121

Hu, S., Shaner, M. R., Beardslee, J. A., Lichterman, M., Brunschwig, B. S., and Lewis, N. S. (2014). Amorphous TiO 2 Coatings Stabilize Si, GaAs, and GaP Photoanodes for Efficient Water Oxidation. Science 344, 1005-1009. doi:10.1126/science. 1251428

Iatsunskyi, I., Kempiński, M., Jancelewicz, M., Załęski, K., Jurga, S., and Smyntyna, V. (2015). Structural and XPS Characterization of ALD Al2O3 Coated Porous Silicon. Vacuum 113, 52-58. doi:10.1016/j.vacuum.2014.12.015

Jiya, I. N., Gurusinghe, N., and Gouws, R. (2018). Electrical Circuit Modelling of Double Layer Capacitors for Power Electronics and Energy Storage Applications: A Review. Electronics 7. doi:10.3390/electronics7110268

Jung, J.-Y., Yu, J.-Y., and Lee, J.-H. (2018a). Dynamic Photoelectrochemical Device Using an Electrolyte-Permeable $\mathrm{NiOx} / \mathrm{SiO} / \mathrm{Si}$ Photocathode with an OpenCircuit Potential of 0.75 V. ACS Appl. Mater. Inter. 10, 7955-7962. doi:10.1021/ acsami.7b16918

Jung, J. Y., Yu, J. Y., Yoon, S., Yoo, B., and Lee, J. H. (2018b). A Photoelectrochemical Device with Dynamic Interface Energetics: Understanding of Structural and Physical Specificities and Improvement of Performance and Stability. Adv. Sust. Syst. 2. doi:10.1002/adsu.201800083

Kalanur, S. S., Singh, R., and Seo, H. (2021). Enhanced Solar Water Splitting of an Ideally Doped and Work Function Tuned \{002\} Oriented One-Dimensional WO3 with Nanoscale Surface Charge Mapping Insights. Appl. Catal. B-Environmental 295. doi:10.1016/j.apcatb.2021.120269

Kats, M. A., Blanchard, R., Ramanathan, S., and Capasso, F. (2014). Thin-film Interference in Lossy, Ultra-thin Layers. Opt. Photon. News 25, 40-47. doi:10.1364/opn.25.1.000040

Kautek, W., Gerischer, H., and Tributsch, H. (1980). The Role of Carrier Diffusion and Indirect Optical Transitions in the Photoelectrochemical Behavior of Layer Type d-Band Semiconductors. J. Electrochem. Soc. 127, 2471-2478. doi:10.1149/ 1.2129499

Kawde, A., Annamalai, A., Sellstedt, A., Uhlig, J., Wågberg, T., Glatzel, P., et al. (2020). More Than protection: the Function of $\mathrm{TiO} 2$ Interlayers in Hematite Functionalized Si Photoanodes. Phys. Chem. Chem. Phys. 22, 28459-28467. doi: $10.1039 / \mathrm{d} 0 \mathrm{cp} 04280 \mathrm{c}$

Kay, A., and Grätzel, M. (2002). Dye-Sensitized Core-Shell Nanocrystals: Improved Efficiency of Mesoporous Tin Oxide Electrodes Coated with a Thin Layer of an Insulating Oxide. Chem. Mater. 14, 2930-2935. doi:10.1021/cm0115968

Klein, A., Mayer, T., Thissen, A., and Jaegermann, W. (2008). Photoelectron Spectroscopy in Materials Science and Physical Chemistry. Sci. Phys. Chem. Bunsen-magazin 10, 124-139.

Laskowski, F. A. L., Oener, S. Z., Nellist, M. R., Gordon, A. M., Bain, D. C., Fehrs, J. L., et al. (2020). Nanoscale Semiconductor/catalyst Interfaces in Photoelectrochemistry. Nat. Mater. 19, 69, 76-+.doi:10.1038/s41563-0190488-z

Le Formal, F., Tétreault, N., Cornuz, M., Moehl, T., Grätzel, M., and Sivula, K. (2011). Passivating Surface States on Water Splitting Hematite Photoanodes with Alumina Overlayers. Chem. Sci. 2, 737-743. doi:10.1039/c0sc00578a
Li, J., Solanki, D., Zhu, Q., Shen, X., Callander, G., Kim, J., et al. (2021). Microstructural Origin of Selective Water Oxidation to Hydrogen Peroxide at Low Overpotentials: a Study on Mn-Alloyed TiO2. J. Mater. Chem. A. 9, 18498-18505. doi:10.1039/d1ta05451a

Lichterman, M. F., Hu, S., Richter, M. H., Crumlin, E. J., Axnanda, S., Favaro, M., et al. (2015). Direct Observation of the Energetics at a Semiconductor/liquid junction by Operando X-ray Photoelectron Spectroscopy. Energy Environ. Sci. 8, 2409-2416. doi:10.1039/c5ee01014d

Lin, C.-P., Chen, H., Nakaruk, A., Koshy, P., and Sorrell, C. C. (2013). Effect of Annealing Temperature on the Photocatalytic Activity of TiO2 Thin Films. Energ. Proced. 34, 627-636. doi:10.1016/j.egypro.2013.06.794

Lin, F., and Boettcher, S. W. (2014). Adaptive Semiconductor/electrocatalyst Junctions in Water-Splitting Photoanodes. Nat. Mater. 13, 81-86. doi:10.1038/nmat3811

Liu, H., Li, W., Shen, D., Zhao, D., and Wang, G. (2015). Graphitic Carbon Conformal Coating of Mesoporous TiO2 Hollow Spheres for HighPerformance Lithium Ion Battery Anodes. J. Am. Chem. Soc. 137, 13161-13166. doi:10.1021/jacs.5b08743

Makula, P., Pacia, M., and Macyk, W. (2018). How to Correctly Determine the Band Gap Energy of Modified Semiconductor Photocatalysts Based on UV-Vis Spectra. J. Phys. Chem. Lett. 9, 6814-6817.

Moehl, T., Suh, J., Sévery, L., Wick-Joliat, R., and Tilley, S. D. (2017). Investigation of (Leaky) ALD TiO2 Protection Layers for Water-Splitting Photoelectrodes. ACS Appl. Mater. Inter. 9, 43614-43622. doi:10.1021/acsami.7b12564

Mohsin, A. S. M., Mobashera, M., Malik, A., Rubaiat, M., and Islam, M. (2020). Light Trapping in Thin-Film Solar Cell to Enhance the Absorption Efficiency Using FDTD Simulation. J. Opt. 49, 523-532. doi:10.1007/s12596-020-00656-w

Nandjou, F., and Haussener, S. (2019). Kinetic Competition between WaterSplitting and Photocorrosion Reactions in Photoelectrochemical Devices. Chemsuschem 12, 1984-1994. doi:10.1002/cssc.201802558

Nellist, M. R., Laskowski, F. A. L., Lin, F., Mills, T. J., and Boettcher, S. W. (2016). Semiconductor-Electrocatalyst Interfaces: Theory, Experiment, and Applications in Photoelectrochemical Water Splitting. Acc. Chem. Res. 49, 733-740. doi:10.1021/acs.accounts.6b00001

Nellist, M. R., Laskowski, F. A. L., Qiu, J., Hajibabaei, H., Sivula, K., Hamann, T. W., et al. (2018). Potential-sensing Electrochemical Atomic Force Microscopy for in Operando Analysis of Water-Splitting Catalysts and Interfaces. Nat. Energ. 3, 46-52. doi:10.1038/s41560-017-0048-1

Nichols, M. T., Li, W., Pei, D., Antonelli, G. A., Lin, Q., Banna, S., et al. (2014). Measurement of Bandgap Energies in Low-K Organosilicates. J. Appl. Phys. 115. doi:10.1063/1.4867644

Nunez, P., Richter, M. H., Piercy, B. D., Roske, C. W., Cabán-Acevedo, M., Losego, M. D., et al. (2019). Characterization of Electronic Transport through Amorphous TiO2 Produced by Atomic-Layer Deposition. The J. Phys. Chem. C. doi:10.1021/acs.jpcc.9b04434

Pan, Z., Röhr, J. A., Ye, Z., Fishman, Z. S., Zhu, Q., Shen, X., et al. (2019). Elucidating Charge Separation in Particulate Photocatalysts Using Nearly Intrinsic Semiconductors with Small Asymmetric Band Bending. Sust. Energ. Fuels 3, 850-864. doi:10.1039/c9se00036d

Paracchino, A., Mathews, N., Hisatomi, T., Stefik, M., Tilley, S. D., and Grätzel, M. (2012). Ultrathin Films on Copper(I) Oxide Water Splitting Photocathodes: a Study on Performance and Stability. Energ. Environ. Sci. 5, 8673-8681. doi:10.1039/c2ee22063f

Park, N.-G., and Zhu, K. (2020). Scalable Fabrication and Coating Methods for Perovskite Solar Cells and Solar Modules. Nat. Rev. Mater. 5, 333-350. doi:10.1038/s41578-019-0176-2

Pastukhova, N., Mavric, A., and Li, Y. B. (2021). Atomic Layer Deposition for the Photoelectrochemical Applications. Adv. Mater. Inter. 8. doi:10.1002/ admi.202002100

Pishgar, S., Strain, J. M., Gulati, S., Sumanasekera, G., Gupta, G., and Spurgeon, J. M. (2019). Investigation of the Photocorrosion of N-GaP Photoanodes in Acid with In Situ UV-Vis Spectroscopy. J. Mater. Chem. A. 7, 25377-25388. doi:10.1039/c9ta10106c

Plana, D., Humphrey, J. J. L., Bradley, K. A., Celorrio, V., and Fermín, D. J. (2013). Charge Transport across High Surface Area Metal/Diamond Nanostructured Composites. ACS Appl. Mater. Inter. 5, 2985-2990. doi:10.1021/am302397p

Richter, M. H., Cheng, W.-H., Crumlin, E. J., Drisdell, W. S., Atwater, H. A., Schmeisser, D., et al. (2021). X-ray Photoelectron Spectroscopy and Resonant 
X-ray Spectroscopy Investigations of Interactions between Thin Metal Catalyst Films and Amorphous Titanium Dioxide Photoelectrode Protection Layers. Chem. Mater. 33, 1265-1275. doi:10.1021/acs.chemmater.0c04043

Roest, A. L., Kelly, J. J., Vanmaekelbergh, D., and Meulenkamp, E. A. (2002). Staircase in the Electron Mobility of a $\mathrm{ZnO}$ Quantum Dot Assembly Due to Shell Filling. Phys. Rev. Lett. 89, 036801. doi:10.1103/PhysRevLett.89.036801

Ros, C., Carretero, N. M., David, J., Arbiol, J., Andreu, T., and Morante, J. R. (2019). Insight into the Degradation Mechanisms of Atomic Layer Deposited TiO2 as Photoanode Protective Layer. ACS Appl. Mater. Inter. 11, 29725-29735. doi:10.1021/acsami.9b05724

Scheuermann, A. G., Lawrence, J. P., Kemp, K. W., Ito, T., Walsh, A., Chidsey, C. E. D., et al. (2016). Design Principles for Maximizing Photovoltage in MetalOxide-Protected Water-Splitting Photoanodes. Nat. Mater 15, 99, 105+.doi:10.1038/nmat4451

Scheuermann, A. G., Prange, J. D., Gunji, M., Chidsey, C. E. D., and Mcintyre, P. C. (2013). Effects of Catalyst Material and Atomic Layer Deposited TiO2 Oxide Thickness on the Water Oxidation Performance of Metal-Insulator-Silicon Anodes. Energ. Environ. Sci. 6, 2487-2496. doi:10.1039/c3ee41178h

Shaner, M. R., Hu, S., Sun, K., and Lewis, N. S. (2015). Stabilization of Si Microwire Arrays for Solar-Driven $\mathrm{H} 2 \mathrm{O}$ Oxidation to $\mathrm{O} 2(\mathrm{~g})$ in $1.0 \mathrm{M} \mathrm{KOH}(\mathrm{aq})$ Using Conformal Coatings of Amorphous TiO2. Energ. Environ. Sci. 8, 203-207. doi:10.1039/c4ee03012e

Sharpe, R., Counsell, J., and Bowker, M. (2017). Pd Segregation to the Surface of Au on $\mathrm{Pd}(111)$ and on $\mathrm{Pd} / \mathrm{TiO} 2(110)$. Surf. Sci. 656, 60-65. doi:10.1016/ j.susc.2016.10.005

Shen, X., Yao, M., Sun, K., Zhao, T., He, Y., Chi, C.-Y., et al. (2021). Defect-Tolerant TiO2-Coated and Discretized Photoanodes for $>600 \mathrm{H}$ of Stable Photoelectrochemical Water Oxidation. ACS Energ. Lett. 6, 193-200. doi:10.1021/acsenergylett.0c02521

Siddiqi, G., Luo, Z., Xie, Y., Pan, Z., Zhu, Q., Röhr, J. A., et al. (2018). Stable Water Oxidation in Acid Using Manganese-Modified TiO2 Protective Coatings. ACS Appl. Mater. Inter. 10, 18805-18815. doi:10.1021/acsami.8b05323

Singh, A., Kumari, S., Shrivastav, R., Dass, S., and Satsangi, V. (2008). Iron Doped Nanostructured TiO2 for Photoelectrochemical Generation of Hydrogen. Int. J. Hydrogen Energ. 33, 5363-5368. doi:10.1016/j.ijhydene.2008.07.041

Sun, K., Kuang, Y., Verlage, E., Brunschwig, B. S., Tu, C. W., and Lewis, N. S. (2015a). Sputtered NiOx Films for Stabilization of P+n-InP Photoanodes for Solar-Driven Water Oxidation. Adv. Energ. Mater. 5. doi:10.1002/ aenm.201402276

Sun, K., Mcdowell, M. T., Nielander, A. C., Hu, S., Shaner, M. R., Yang, F., et al. (2015b). Stable Solar-Driven Water Oxidation to O2(g) by Ni-OxideCoated Silicon Photoanodes. J. Phys. Chem. Lett. 6, 592-598. doi:10.1021/jz5026195

Sun, K., Saadi, F. H., Lichterman, M. F., Hale, W. G., Wang, H.-P., Zhou, X., et al. (2015c). Stable Solar-Driven Oxidation of Water by Semiconducting Photoanodes Protected by Transparent Catalytic Nickel Oxide Films. Proc. Natl. Acad. Sci. USA 112, 3612-3617. doi:10.1073/pnas.1423034112

Sze, S. M., and Ng, K. K. (2007). Physics of Semiconductor Devices. Hoboken, NJ: Wiley-Interscience.

Tan, M. X., Laibinis, P. E., Nguyen, S. T., Kesselman, J. M., Stanton, C. E., and Lewis, N. S. (1994). Principles and Applications of Semiconductor Photoelectrochemistry. Prog. Inorg. Chem. 41, 21-144.
Thorne, J. E., Li, S., Du, C., Qin, G., and Wang, D. (2015). Energetics at the Surface of Photoelectrodes and its Influence on the Photoelectrochemical Properties. J. Phys. Chem. Lett. 6, 4083-4088. doi:10.1021/acs.jpclett.5b01372

Vanka, S., Sun, K., Zeng, G., Pham, T. A., Toma, F. M., Ogitsu, T., et al. (2019). Long-term Stability Studies of a Semiconductor Photoelectrode in ThreeElectrode Configuration. J. Mater. Chem. A. 7, 27612-27619. doi:10.1039/ c9ta09926c

Walter, M. G., Warren, E. L., Mckone, J. R., Boettcher, S. W., Mi, Q., Santori, E. A., et al. (2010). Solar Water Splitting Cells. Chem. Rev. 110, 6446-6473. doi: $10.1021 / \mathrm{cr} 1002326$

Wang, H., Turner, J. A., Li, X., and Teeter, G. (2008). Process Modification for Coating SnO2:F on Stainless Steels for PEM Fuel Cell Bipolar Plates. J. Power Sourc. 178, 238-247. doi:10.1016/j.jpowsour.2007.12.010

Wolter, H. (1966). Born,M - Principles of Optics Electromagnetic Theory of Propagation Interference and Diffraction of Light. Z. Angew. Physik 21, 565.

Xu, J., Nagasawa, H., Kanezashi, M., and Tsuru, T. (2018). UV-protective TiO2 Thin Films with High Transparency in Visible Light Region Fabricated via Atmospheric-Pressure Plasma-Enhanced Chemical Vapor Deposition. ACS Appl. Mater. Inter. 10, 42657-42665. doi:10.1021/acsami.8b15572

Yang, H., Bright, J., Kasani, S., Zheng, P., Musho, T., Chen, B., et al. (2019). Metalorganic Framework Coated Titanium Dioxide Nanorod Array P-N Heterojunction Photoanode for Solar Water-Splitting. Nano Res. 12, 643-650. doi:10.1007/s12274-019-2272-4

Yu, Y., Sun, C., Yin, X., Li, J., Cao, S., Zhang, C., et al. (2018). Metastable Intermediates in Amorphous Titanium Oxide: A Hidden Role Leading to Ultra-stable Photoanode Protection. Nano Lett. 18, 5335-5342. doi:10.1021/ acs.nanolett.8b02559

Zhao, T. S., Yanagi, R., Xu, Y. J., He, Y. L., Song, Y. Q., Yang, M. Q., et al. (2021). A Coating Strategy to Achieve Effective Local Charge Separation for Photocatalytic Coevolution. Proc. Natl. Acad. Sci. United States America 118. doi:10.1073/pnas.2023552118

Zheng, J. Y., Zhou, H. J., Zou, Y. Q., Wang, R. L., Lyu, Y. H., Jiang, S. P., et al. (2019). Efficiency and Stability of Narrow-gap Semiconductor-Based Photoelectrodes. Energ. Environ. Sci. 12. doi:10.1039/c9ee00524b

Conflict of Interest: The authors declare that the research was conducted in the absence of any commercial or financial relationships that could be construed as a potential conflict of interest.

Publisher's Note: All claims expressed in this article are solely those of the authors and do not necessarily represent those of their affiliated organizations, or those of the publisher, the editors and the reviewers. Any product that may be evaluated in this article, or claim that may be made by its manufacturer, is not guaranteed or endorsed by the publisher.

Copyright $\odot 2022$ Shen, Yanagi, Solanki, Su, Li, Xiang and Hu. This is an openaccess article distributed under the terms of the Creative Commons Attribution License (CC BY). The use, distribution or reproduction in other forums is permitted, provided the original author(s) and the copyright owner(s) are credited and that the original publication in this journal is cited, in accordance with accepted academic practice. No use, distribution or reproduction is permitted which does not comply with these terms. 